\title{
Single Mossy Fiber Axonal Systems of Human Dentate Granule Cells Studied in Hippocampal Slices from Patients with Temporal Lobe Epilepsy
}

\author{
Masako Isokawa, ${ }^{1}$ Michel F. Levesque, ${ }^{2}$ Thomas L. Babb, ${ }^{1,3}$ and Jerome Engel, Jr., ${ }^{1,3}$ \\ 'Brain Research Institute and Departments of ${ }^{2}$ Neurosurgery and ${ }^{3}$ Neurology, School of Medicine, University of California, \\ Los Angeles, California 90024-1761
}

Previous histological and immunocytochemical studies suggest that reorganization of the dentate granule cell axons, the mossy fibers, can occur in epileptic human hippocampus (Sutula et al., 1989; Houser et al., 1990; Babb et al., 1991) and in animal models of epilepsy (Tauck and Nadler, 1985; Sutula et al., 1988; Cronin et al., 1992). However, neuroanatomical analyses of the trajectory and morphology of reorganized axons are not yet available. The present study was conducted to investigate single dentate granule cell axonal systems in human epileptic hippocampus. Individual mossy fibers were directly visualized by injecting a tracer (biocytin or Lucifer yellow) intracellularly in hippocampal slices prepared from temporal lobes that were surgically removed from patients for treatment of intractable epilepsy. Two major arborization patterns were identified: (1) the parent axons extended to and coursed through the hilus toward CA3, leaving collaterals along their paths in the hilus $(N=$ 19 neurons); (2) in addition to the aforementioned axonal system, collateral(s) branched from the parent axon near the soma and projected to the granule cell layer and molecular layer, forming an aberrant axonal pathway ( $N=9$ neurons). These aberrant collaterals bore large boutons similar to those of the hilar axons and formed extensive plexuses in the granule cell layer and/or in the molecular layer. The summed length of collaterals in the granular/molecular layers was $1110.8 \mu \mathrm{m}$ on average, which was one-fourth of the total summed length of the mossy fibers (3698.5 $\mu \mathrm{m}$ on average). The size of the somata in neurons that had aberrant collaterals was significantly larger than that of neurons without such collaterals $(p<0.025)$. In four cases, filopodium-like fine processes were present near the axon hillock and proximal parts of the parent axon, suggesting that the aberrant collateral formation might be an ongoing process in these tissues. The lack of control slices from normal living human hlppocampus makes it difficult to assess to what extent the

\footnotetext{
Received June 9, 1992; revised Oct. 6, 1992; accepted Oct. 12, 1992.

We thank Dr. F. E. Dudek for his assistance in setting up our brain slice system, Dr. R. S. Fisher for letting us use his fluorescent microscope, Dr. D. M. Finch for helpful comments and editorial assistance, and the members of the Clinical Neurophysiology Project for their clinical expertise. This research was conducted with permission of the UCI.A Human Subjects Protection Committee and in accordance with the guidelines of the Declaration of Helsinki. This work was supported by NIH Grant NS02808.

Correspondence should be addressed to Masako Isokawa, Ph.D., Brain Research Institute, CHS, UCLA, Los Angeles, CA 90024-1761.

Copyright (C) 1993 Society for Neuroscience $0270-6474 / 93 / 131511-12 \$ 05.00 / 0$
}

present findings are epilepsy associated. However, the presence of aberrant mossy fiber collaterals in the hippocampi used in the present study has been confirmed by Timm's staining and/or dynorphin immunohistochemistry in comparison with nonepileptic autopsy material, indicating its relation to epilepsy (Babb et al., 1991, 1992). At present, there seems to be a consensus that the projection of mossy fiber collaterals to the supragranular layer is a rare occurrence in normal rats (Lorento de Nó, 1934; Claiborne et al., 1986; Seress et al., 1991; present study), normal monkeys (Seress et al., 1991), and normal humans (Houser et al., 1990). Thus, we believe that the intracellularly stained aberrant axon collaterals reported here in the supragranular layer likely represent trajectories of reorganized mossy fibers associated with medically intractable temporal lobe epilepsy.

[Key words: intracellular injections, granule cell axons, biocytin, Lucifer yellow, sprouting, filopodia]

Recent histological and immunocytochemical studies on epileptic human hippocampus suggest that the dentate granule cell axons, the mossy fibers, in epileptic hippocampus have a collateral arborization pattern that is different from that of normal mossy fibers in lower mammals and in autopsy control human tissues (Sutula et al., 1989; Houser et al., 1990; Babb et al., 1991). These aberrant collaterals were localized by Timm's staining or dynorphin immunoreactivity as a band of reaction product in the supragranular region, especially in the inner molecular layer of the dentate gyrus. Because the mossy fibers normally cxtend their arbors to the polymorph (hilar) region of the dentate gyrus and CA3 field of the hippocampus proper, but do not project to the molecular layer (Lorento de Nó, 1934; Claiborne et al., 1986), epileptogenic dentate granule cells have been thought to sprout axon collaterals beyond their normal arborization domain. The intensity of this sprouting showed a positive correlation to the severity of seizure activities (Sutula et al., 1989) and the severity of hilar cell loss (Houser et al., 1990). This finding in human epileptic hippocampus was supported by several animal models of epilepsy. The kainate model (Tauck and Nadler, 1985), the kindling model (Sutula et al., 1988), and the pilocarpine model (Mello et al., 1990), all showed a dense band of Timm's staining in the inner molecular layer, which was indicative of reorganized mossy fiber paths. Physiological studies in the kainate model suggested that sprouted fibers could form recurrent excitatory circuits, constituting a possible sub- 
strate of epileptogenicity (Tauck and Nadler, 1985; Cronin et al., 1992). However, cellular anatomy to elucidate the paths and morphology of the sprouted fibers has not been reported in either human epileptic hippocampus or experimental models of epilepsy.

In the present study, we used a method of intracellular injection of tracers (biocytin or Lucifer yellow) into living single dentate granule cells in human epileptic hippocampus maintained in vitro, and directly visualized their axonal morphology and arborization patterns. The advantage of using this method is (1) only a single neuron is stained at any one time per slice, so that all fibers visualized can be considered to originate in the stained neuron. This overcomes the disadvantage of using the Golgi staining, which stains multiple neurons simultaneously and yct capriciously stains only a subpopulation of total fibers. (2) In cases where stained neurons have aberrant fibers, the relationship between the parent axon in the hilus and the aberrant collaterals in the molecular layer can be qualitatively and quantitatively studied. (3) Injecting a tracer in living cells may minimize the possibility of obscuring fine axonal branches that might occur in autopsy or biopsy tissues prepared for pathological examinations.

From the nature of this study, which uses living human hippocampal specimens, our findings cannot be directly compared to normal controls that would be obtained from disease-free living human hippocampus. Ethical considerations preclude the use of such tissue. In the present study, we made the following efforts to establish alternative control comparisons in interpreting our data. First, we compared the present human hippocampal slice data with normal rat hippocampal slice data. The experiments were performed using the same techniqucs, equipment, and slice chambers in both groups, using the same experimental protocol. This method provides a certain degree of support in contrasting differences in cell morphology between an epileptic condition and a normal condition. It also rules out some technical problems that might arise in interpreting human results when we completely relied on others for control data. However, this method risks underestimating species differences in dentate granule cell morphology. For example, basal dendrites have been identified as normal dendritic morphology of dentate granule cells in monkeys and humans (Seress and Mrzljak, 1987), but not in rats (Seress and Pokorny, 1981; Desmond and Levy, 1982). Thus, careful comparative studies in mossy fiber morphology in the phylogenetic tree cannot be neglected in interpreting our data. At present, there seems to be a consensus that the presence of mossy fiber collaterals in the molecular layer is a rare occurrence in normal rats (Seress et al., 1991), normal monkeys (Seress et al., 1991), and normal humans (Houser et al., 1990). Second, the present findings of aberrant collaterals in the supragranule layer are supported by neuropathological examinations that assess the location of mossy fibers in the human epileptic hippocampus. These examinations were made in the hippocampi used in the present study. The adjacent blocks of the hippocampus of the same individual patients used in the present study were examined for (1) cell density and (2) Timm's staining and/or dynorphin immunoreactivity by neuroanatomists in the UCLA Epilepsy Surgery Program. These neuropathological results were compared with normal autopsy specimens, which were disease free. The presence of cell loss and mossy fiber reorganization (i.e., collaterals in the supragranular layer) in these patients has been attributed to temporal lobe epilepsy (Babb et al., 1991, 1992).

\section{Materials and Methods}

Patient selection. Hippocampal slices were obtained from 23 patients who had medically intractable temporal lobe epilepsy and entered the epilepsy surgery program for treatment of their seizure disorders. In all of them, there was evidence of a medial temporal origin of epileptogenic discharges. Patient selection was based on a series of evaluations that included EEG monitoring, neurological and cognitive testing, positron emission tomography, magnetic resonance imaging, and depth electrode implants (Engel, 1987). Neuroanatomical examinations of the surgically removed specimens included cell counts to assess neuronal death and hippocampal sclerosis in epileptogenic regions (Babb et al., 1984). All of these were performed on cvery hippocampal specimen as part of routine examinations for the identification of epileptic focal pathology. The results of pathological examinations were made available from the data base of the UCLA Epilepsy Surgery Program.

Brain tissues. The hippocampal tissue for slice preparation was obtained directly from neurosurgeons at the University of California at Los Angeles. The resected hippocampus was immediately immersed in oxygen-containing ice-cold artificial cerebrospinal fluid consisting of (in mm) $124 \mathrm{NaCl}, 3 \mathrm{KCl}, 2.4 \mathrm{CaCl}_{2}, 26 \mathrm{NaHCO}_{3}, 1.3 \mathrm{MgSO}_{4}, 1.24 \mathrm{NaH}_{2} \mathrm{PO}_{4}$, and 10 glucose, $\mathrm{pH}$ 7.4. Slices were cut perpendicular to the anteriorposterior axis of the hippocampus at a thickness of $500 \mu \mathrm{m}$ (Vibroslicer, World Precision Instruments), and placed on the ramp of a liquid-gas interface recording chamber (Haas et al., 1979) at $34 \pm 1^{\circ} \mathrm{C}$. As a control comparison, normal rat hippocampal slices were prepared $(10$ males, 150-200 gm; Charles River). The dentate granule cells were injected with the same tracers and with the same experimental protocols using our human brain slice chamber. This procedure provided (1) technical confirmation on intracellular dye filling in human epileptic neurons and (2) a guideline for criteria in selecting neurons with satisfactory tracer injections.

Intracellular injection of tracers. Either biocytin (Sigma) or Lucifer yellow (in lithium chloride salt, Sigma) was used. Intracellular electrodes were prepared from borosilicate glass pipettes, and filled with $2 \mathrm{M}$ potassium acetate with $2 \%$ biocytin (Horikawa and Armstrong, 1988) or $0.1 \mathrm{M}$ lithium chloride with 5\% Lucifer yellow (Stewart, 1978). Electrodes were positioned at the dentate granule cell layer and lowered by a hydraulic micromanipulator (Narishige, WR-88). The penetration of a cell was determined by measuring the resting membrane potential ( 50 to $65 \mathrm{mV}$ ). In those cases in which cells were held $30 \mathrm{~min}$ or longer with biocytin-filled electrode, biocytin was allowed to diffuse into the intracellular space passively. Otherwise, direct negative current pulses (1-2 nA; $300 \mathrm{msec}$ in duration; $0.5 \mathrm{~Hz})$ were applied through an electrode for several minutes to inject the tracers. Lucifer yellow-injected slices were fixed by $4 \%$ paraformaldehyde and mounted on glass slides. The injected cells were examined and photographed under a fluorescence microscope (Nikon Microphoto-FXA). Biocytin-injected slices were first fixed by $4 \%$ paraformaldehyde, and then sectioned into $30 \mu \mathrm{m}$ thickness and processed according to the method of Horikawa and Armstrong (1988). Biocytin-filled neurons were detected by a reaction product of horseradish peroxidase and diaminobenzidine. Cell morphology was examined and photographed under a light microscope (Zeiss Universal microscope), and traced by using a camera lucida. There were no noticeable differences between Lucifer yellow-injected neurons and biocytin-injected neurons in visualizing cell morphology for the purpose of the present study, so the data were pooled for the analysis. When measurements were made for quantification, they were done on camera lucida drawings or directly from the processed specimens by light microscopy.

\section{Results}

Seventy-two neurons in 23 hippocampi from 23 patients were intracellularly injected and visualized with either biocytin or Lucifer yellow. Among them, 19 neurons (11 neurons with biocytin and 8 neurons with Lucifer yellow) in 10 patients (15-44 years of age; 2 males and 8 females) showed satisfactory injection of the tracers and were selected for analysis. Selection criteria were based on (1) the extent of stained mossy fibers that showed the arborization beyond the parent axon and primary collaterals in the hilus, and (2) the presence of clearly visible varicosities along the fibers. When neurons showed densely stained axonal arbors, they also showed discretely stained somata and well- 

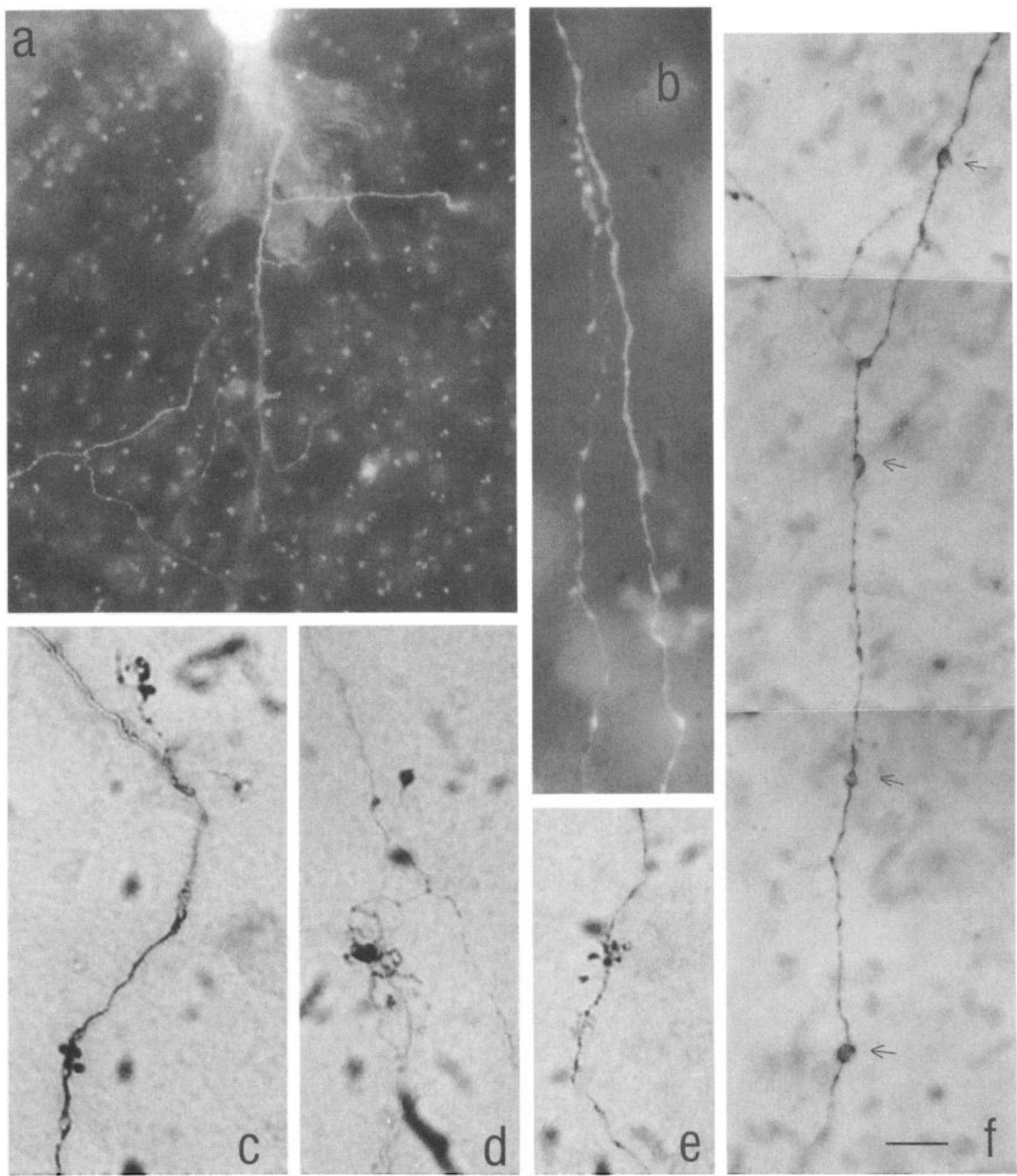

Figure 1. a, Dentate granule cell axons (the mossy fibers) were stained in the hilus by intracellular injection of Lucifer yellow. The vertically oriented parent axon extruded many branching collaterals. The soma and basal dendrites are seen at the top of the photomicrograph (out of focus). $b$, The fusiform-shaped varicosities were observed along the mossy fibers (stained by Lucifer yellow). $c$ and $d$, The large, irregularly shaped varicosities (biocytin). $e$, A floweret-like terminal was observed along the fibers. Fusiform-shaped varicosities were also stained. $f$, Axonal lacunae (arrows) were present in addition to varicosities. These lacunae were larger in size than varicosities, and only the contour was stained. Scale bar: $a, 30 \mu \mathrm{m} ; b, 13.5 \mu \mathrm{m} ; c-e, 12 \mu \mathrm{m} ; f, 18 \mu \mathrm{m}$.

developed dendritic arbors with detailed spine structures as reported elsewhere (Isokawa and Levesque, 1991).

\section{Mossy fibers in the hilus}

Figure $1 a$ shows a photomicrograph of mossy fiber arbors in the hilus stained by Lucifer yellow. In this neuron, axons showed a vertically oriented arborization pattern. The collaterals branched out from both proximal and distal parts of the parent axon. The vertically oriented arborization pattern was observed in eight neurons. In the remaining neurons $(N=11)$, the arborization pattern was predominantly oriented horizontally, showing well-developed collaterals covering a wide range of the hilar region. Camera lucida drawings of horizontal and vertical arbors are shown in Figure 2, $A$ and $B$, respectively. An example of a normal rat mossy fiber is shown in Figure $2 C$ as a comparison. In the human mossy fibers of the present group of specimens, there were a greater number of local collaterals compared with normal rats, and these collaterals formed complex arborization patterns. In six neurons, densely formed plexuses were observed in some collaterals. An example is shown in Figure $4 C$ (arrow 5).

Varicosities were present along the axon collaterals. They were densely stained by both biocytin and Lucifer yellow. Two different shapes were identified: the fusiform shape with a length 


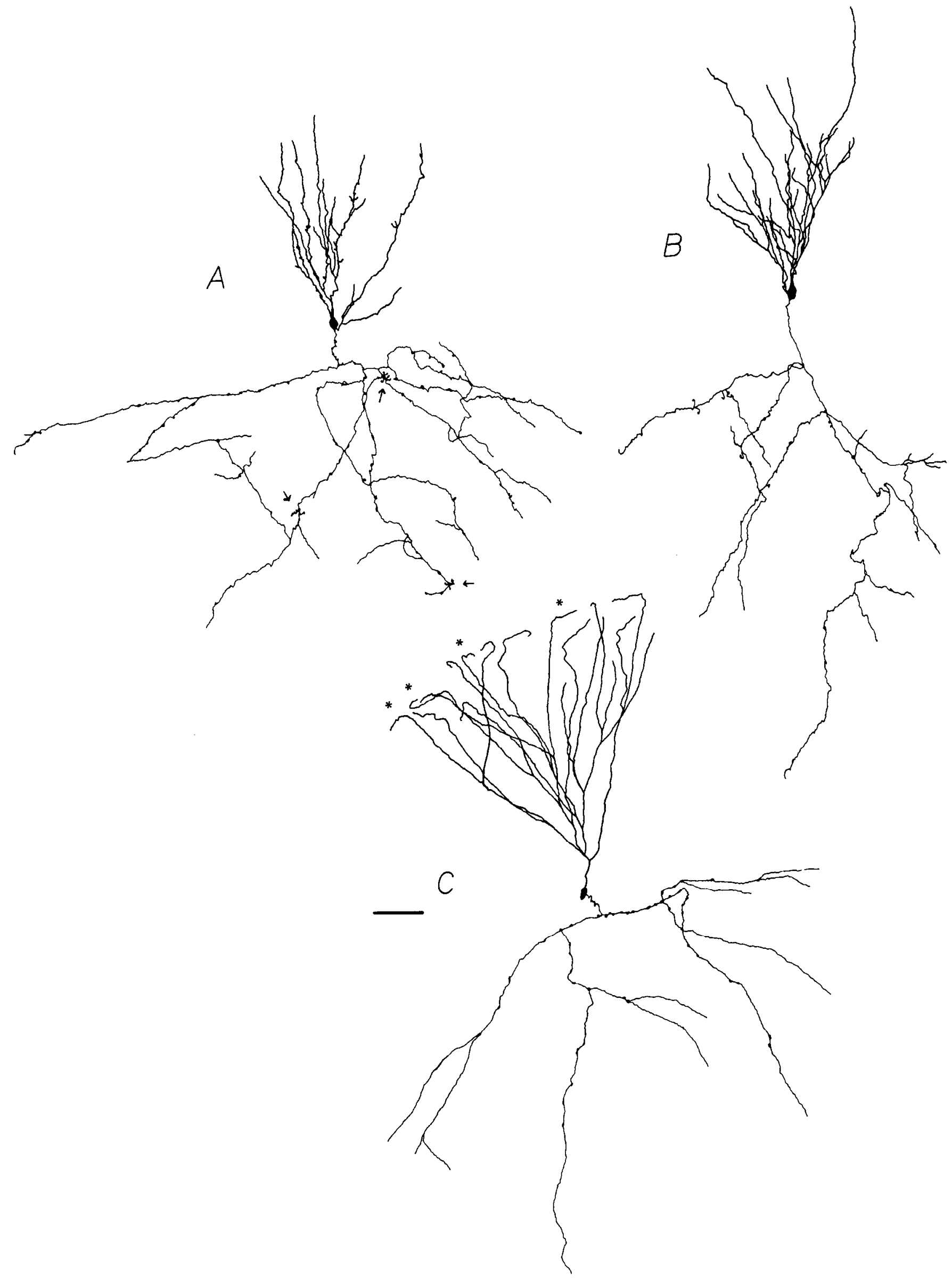


of 2-5 $\mu \mathrm{m}$ (Fig. $1 b$ ) and the irregular shape, $4-5 \mu \mathrm{m}$ in size (Fig. $1 c, d)$. Fusiform varicosities were regularly present along the fibers at approximately $7-18 \mu \mathrm{m}$ intervals in most cases, but occasionally at $35-50 \mu \mathrm{m}$ intervals. The irregular varicosities were larger in size and present along the fibers and at endings. They were more sparsely located than the fusiform varicosities. The locations of the large, irregular varicosities in axonal arbors were plotted in the camera lucida drawings whenever they were clearly visible with low magnification (see Figs. 2, 4). These two types of varicosities were also common in the control rat mossy fibers. The locations of the large, irregular varicosities in one of our control rat dentate granule cells are depicted in Figure $2 C$. In addition, in human mossy fibers, round varicosities were observed at branching points. The diameter of these varicosities were 2-3 $\mu \mathrm{m}$. In two neurons, one stained with biocytin and the other stained with Lucifer yellow, terminals shaped like a floweret were observed. These terminals looked like five to eight round varicosities with short stems extruding from the same spot of the collateral and forming a blooming flower petal appearance (Fig. 1e). Some of the irregular varicosities, especially larger ones, could be irregularly aggregated flowerets. In addition to the varicosities, lacunae were observed as large swellings along the fibers (arrows in Fig. 1f). The shape of the lacunae was mostly round, and the size was considerably larger $(5-7 \mu \mathrm{m}$ in diameter) than that of the varicosities. In contrast to the varicosities, which were stained dark, the inside of lacunae were pale when they were labeled with biocytin or Lucifer yellow.

\section{Axon collaterals in the granule and molecular layers}

In addition to arbors in the hilus, mossy fiber collaterals were observed in the dentate granule cell layer and/or molecular layer in nine human dentate granule cells. It was a common feature for these aberrant collaterals to branch out from the parent axon at a proximal point, sometimes even before the initial branching point of the hilar collaterals. A representative example of a biocytin-stained aberrant mossy fiber collateral is shown in Figure $3 a$. The neuron in this figure showed two collaterals that extended to the molecular layer by branching out from the parent axon near the soma. These aberrant collaterals passed through the granule layer and formed a dense plexus with many terminal boutons in the molecular layer (Fig. $3 b, c$ ). One collateral extended farther beyond the plexus and reached its own dendritic domain (Fig. 3a, upper left). A camera lucida drawing of this neuron is shown in Figure $4 A$. The aberrant collateral plexus is indicated by arrow 1. A dense plexus and intensive collateral formation in the molecular layer were observed in two of nine neurons. In another four of nine neurons, mossy fiber collaterals were mostly limited to the granule cell layer (Fig. 4B). They formed dense plexuses in the granule cell layer, and the somata were surrounded by fine axon collaterals (arrow 2 in Fig. $4 B$ ). Some of them extended to the molecular layer (arrow 3 in Fig. $4 B$ ). As the somata of these neurons were located in the granule cell layer, the presence of aberrant collaterals around the somata was not due to granule cell dispersion in which granule cell somata were dislocated to the molecular layer (Houser, 1990). Finally, in the remaining three neurons, single collaterals were identified in the molecular layer. We did not observe plexuses in these collaterals. An example is shown in Figure $3 d$. In this photomicrograph, a single collateral branched out from the parent axon at a proximal level and extended back towards the molecular layer. Figure $4 C$ shows another example of a single aberrant collateral in the molecular layer. In this case, the entire length of the collateral could not be traced; thus, a branching point of this collateral from the parent axon was not identified. No plexuses were observed in this collateral.

As shown in Figure $3 b$, aberrant collaterals bore large irregular varicosities. They were especially packed in plexuses. Fusiformshaped varicosities were also present (Fig. 3c). The sizes of these varicosities were not different from those observed in the hilus. Although, on occasion, large and clearly visible lacunae were present near branching points of aberrant collaterals (Fig. $3 d$ ), there were rarely lacunae observed in the collaterals located in the molecular layer. In addition, filopodium-like fine processes were observed in four neurons. These processes were located near the axon hillock (Fig. $5 A$ ) and at the proximal part of the parent axon below the hillock (Fig. $5 B$ ). In $75 \%$ of the cases in which neurons showed these filopodia (three out of four neurons), they also showed mossy fiber collaterals in either the dentate gyrus granular layer or the molecular layer. We observed terminal enlargement in some of these filopodia, which could be a putative growth cone. An example is shown in Figure 5.

The extent of aberrant collaterals in individual neurons was estimated quantitatively by calculating the summed lengths of axon branches in the dentate gyrus. Due to a technical limitation of bleaching Lucifer yellow during the observation, this calculation was done only in biocytin-filled neurons on camera lucida drawings. The average length of summed mossy fibers in the hilus was $3698.5 \mu \mathrm{m} \pm 461.9 \mathrm{SEM}$ in 10 neurons. On the other hand, the average length of summed mossy fibers in the granule layer and molecular layer was $1110.8 \mu \mathrm{m} \pm 345.9 \mathrm{SEM}$ in four neurons. This indicates that, on average, a quarter of the total mossy fiber lengths became aberrant collaterals (Table 1). In addition, the size of somata in neurons with and without abcrrant collatcrals was compared. This was done in all neurons stained by biocytin or Lucifer yellow by measuring the longer and shorter dimensions of ovoid-shaped somata. As shown in Table 2, the soma size was significantly greater in neurons with aberrant collaterals than in neurons without those collaterals ( $p$ $<0.025, t=2.19$; the longer dimension is called length and the shorter dimension is called width in the table).

\section{Discussion}

By using an intracellular labeling technique, we have elucidated details of morphology and arborization patterns of the mossy fibers in epileptic human hippocampus. Extensively ramified axon collaterals of dentate granule cells were visualized, as previously shown by Golgi studies (Scheibel et al., 1974). However, in addition to cell morphology with Golgi-like appearance, our

Figure 2. Camera lucida drawings of two human dentate granule cells $(A$ and $B)$ and a rat dentate granule cell $(C)$. $A, A$ well-developed axon arbor covered a wide horizontal domain in the hilus. The large, irregular varicosities are illustrated along the fibers whenever they were obvious with low magnification. Floweret-like terminal boutons were observed in proximal and distal branches (arrows). B, A vertically oriented parent axon was accompanied by the collaterals that branched out from various portions of the parent axon. $C$, A dentate granule cell in normal rat hippocampus. A well-developed axon arbor and clearly identifiable varicosities were observed. The distal ends of most of the dendrites were clearly visualized as bending tips staying at the outer edge of the molecular layer without crossing the hippocampal fissure (asterisks). These features indicate that the dye was satisfactorily transported throughout the dendrites. Scale bar: $A$ and $B, 100 \mu \mathrm{m} ; C, 45.5 \mu \mathrm{m}$. 


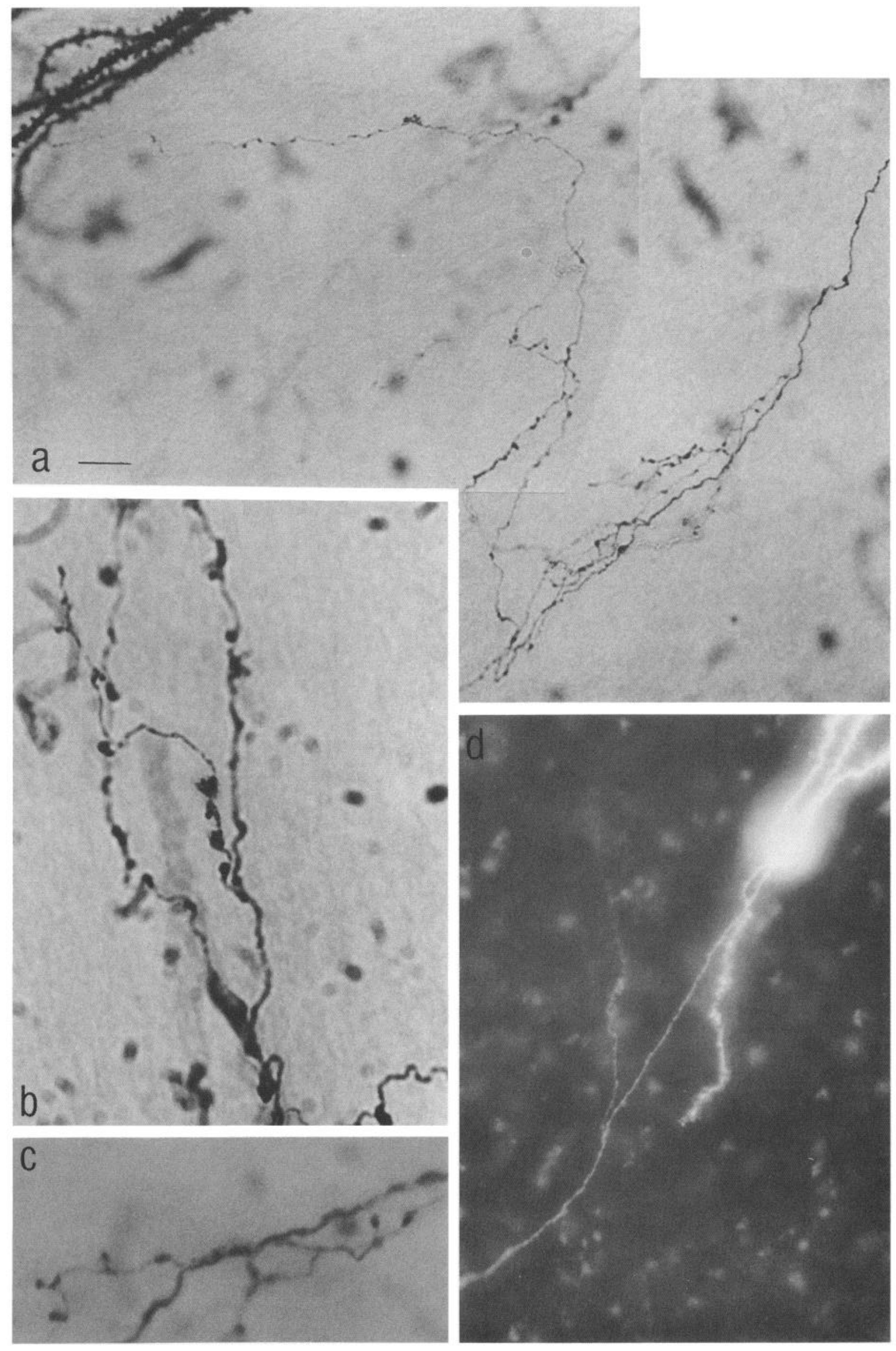


Table 1. Lengths of summed axon branches (mossy fibers) in dentate gyrus

\begin{tabular}{llrl} 
Locations & Average \pm SEM & Range & \\
\hline Hilus & $3698.5 \mu \mathrm{m} \pm 461.9 \mathrm{SEM}$ & $2193-6496 \mu \mathrm{m}$ & $(N=10)$ \\
Dentate gyrus (GL and ML) & $1110.8 \mu \mathrm{m} \pm 345.9 \mathrm{SEM}$ & $620-2133 \mu \mathrm{m}$ & $(N=4)$ \\
\hline
\end{tabular}

Calculations were done on neurons stained by biocytin only. GL, granule cell layer; ML, molecular layer.

\begin{tabular}{|c|c|c|}
\hline Cells & \multicolumn{2}{|c|}{ [Average length $(L) \pm \mathrm{SEM}] \times[$ Average width $(W) \pm \mathrm{SEM}]$} \\
\hline With aberrant collaterals & {$[25.0(L) \pm 2.1] \times[14.0(W) \pm 1.3] \mu \mathrm{m}^{*}$} & $(N=9)$ \\
\hline Without aberrant collaterals & {$[19.1(L) \pm 1.6] \times[12.8(W) \pm 1.4] \mu \mathrm{m}$} & $(N=10)$ \\
\hline
\end{tabular}

* Measurement was done in all the neurons stained by either biocytin or Lucifer yellow. The difference was significant by Student's $t$ test at the level of $p<0.025, t=2.19$.

Table 3. Relationships between mossy fiber arborization patterns and other neuropathological findings in the dentate gyrus

\begin{tabular}{|c|c|c|c|c|c|c|c|c|c|c|c|}
\hline \multirow{2}{*}{$\begin{array}{l}\text { Pa- } \\
\text { tient }\end{array}$} & \multirow{2}{*}{$\begin{array}{l}\text { Age } \\
(\mathrm{yr})\end{array}$} & \multirow[b]{2}{*}{ Sex } & \multirow{2}{*}{$\begin{array}{l}\text { Age at } \\
\text { seizure onset } \\
(y r)\end{array}$} & \multirow[b]{2}{*}{ Hilar cell loss } & \multirow{2}{*}{$\begin{array}{l}\text { Granule layer } \\
\text { cell loss }\end{array}$} & \multirow{2}{*}{$\begin{array}{l}\text { Mossy fiber } \\
\text { reorganization } \\
\text { detected by: }\end{array}$} & \multirow{2}{*}{$\begin{array}{l}\text { Intra- } \\
\text { cellularly } \\
\text { stained } \\
\text { neurons }\end{array}$} & \multicolumn{3}{|c|}{ Mossy fibers in: } & \multirow[b]{2}{*}{ Filopodia } \\
\hline & & & & & & & & Hilus & GL & ML & \\
\hline \multirow[t]{2}{*}{ A } & 36 & $\mathrm{~F}$ & 21 & $0 \%$ & $21-32 \%$ & Timm's staining & \#1 (B) & $\mathrm{x}(\mathrm{pl})$ & & & \\
\hline & & & & & $(27 \pm 5.5 \mathrm{SEM})$ & & \#2 (B) & $\mathbf{x}$ & & & \\
\hline \multirow[t]{3}{*}{ B } & 24 & $\mathrm{~F}$ & 4 & $5 \%$ & $38-46 \%$ & Timm's staining & \#1 (B) & $\mathbf{x}$ & & & \\
\hline & & & & & $(42 \pm 4.0 \mathrm{SEM})$ & & $\# 2$ (B) & $\mathbf{x}$ & & & \\
\hline & & & & & & & \#3 (B) & $\mathrm{x}(\mathrm{pl})$ & & & \\
\hline \multirow{2}{*}{ C } & 44 & $\mathbf{M}$ & 31 & $0 \%$ & $35-48 \%$ & Dynorphin staining & \#1 (LY) & $\mathbf{x}$ & $\mathrm{x}(\mathrm{pl})$ & & $\mathbf{x}$ \\
\hline & & & & & $(42 \pm 6.5 \mathrm{SEM})$ & & & & & & \\
\hline \multirow[t]{2}{*}{ D } & 29 & $\mathbf{F}$ & 2.5 & $50 \%$ & $42-46 \%$ & Dynorphin staining & \#1 (LY) & $\mathrm{x}$ & & & \\
\hline & & & $\begin{array}{l}\text { (convulsion, } \\
10 \text { months) }\end{array}$ & & $(44 \pm 2.0 \mathrm{SEM})$ & & & & & & \\
\hline \multirow[t]{2}{*}{ E } & 38 & $\mathbf{F}$ & 13 & $43-50 \%$ & $30-52 \%$ & Dynorphin staining & \#1 (LY) & $\mathbf{x}$ & & & $\mathbf{x}$ \\
\hline & & & & $(4.7 \pm 3.5 \mathrm{SEM})$ & $(38 \pm 5.1 \mathrm{SEM})$ & & \#2 (LY) & $\mathbf{x}$ & & $\mathbf{x}$ & $\mathbf{x}$ \\
\hline \multirow[t]{2}{*}{$F$} & 19 & $F$ & 1.2 & $65 \%$ & $50-51 \%$ & Dynorphin staining & $\# 1(\mathrm{LY})$ & $\mathbf{x}$ & & & \\
\hline & & & & & $(51 \pm 0.5 \mathrm{SEM})$ & & $\# 2$ (B) & $\mathrm{x}(\mathrm{pl})$ & & & \\
\hline \multirow[t]{4}{*}{$\mathbf{G}$} & 29 & $\mathrm{~F}$ & $5 \mathrm{~d}$ & $75 \%$ & $58-66 \%$ & Timm's and dynorphin & \#1 (B) & $\mathbf{X}$ & & & \\
\hline & & & (forceps & & $(62 \pm 4.0 \mathrm{SEM})$ & staining & $\# 2$ (B) & $\mathrm{x}(\mathrm{pl})$ & & $\mathrm{x}(\mathrm{pl})$ & \\
\hline & & & delivery, & & & & \#3 (B) & $\mathrm{x}(\mathrm{pl})$ & & $\mathrm{x}(\mathrm{pl})$ & \\
\hline & & & $\begin{array}{l}\text { seizure at } \\
\text { birth) }\end{array}$ & & & & & & & $\mathbf{x}$ & \\
\hline \multirow[t]{2}{*}{$\mathbf{H}$} & 25 & $\mathbf{M}$ & 10 & $24-75 \%$ & $53-78 \%$ & Dynorphin staining & \#1 (B) & $\mathrm{x}$ & $\mathrm{x}(\mathrm{pl})$ & & \\
\hline & & & & $(50 \pm 25.5 \mathrm{SEM})$ & $(62 \pm 5.6 \mathrm{SEM})$ & & \#2 (LY) & $\mathbf{x}$ & & $\mathbf{x}$ & $\mathbf{x}$ \\
\hline \multirow[t]{2}{*}{ I } & 15 & $\mathbf{F}$ & 3 & $75 \%$ & $68-73 \%$ & No data & \#1 (LY) & $\mathbf{x}$ & & & \\
\hline & & & $\begin{array}{l}\text { (febrile } \\
\text { seizure, } \\
1.3 \mathrm{yr} \text { ) }\end{array}$ & & $(62 \pm 9.0 \mathrm{SEM})$ & & $\# 2(\mathrm{LY})$ & $\mathbf{x}$ & $\mathbf{x}$ & & \\
\hline $\mathbf{J}$ & 30 & $\mathrm{~F}$ & 1.5 & No data & No data & No data & \#1 (B) & $\mathrm{x}(\mathrm{pl})$ & $\mathrm{x}(\mathrm{pl})$ & & \\
\hline
\end{tabular}

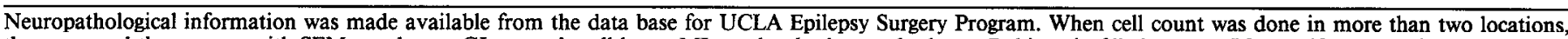
the range and the average with SEM are shown. GL, granule cell layer; ML, molecular layer; pl, plexus; B, biocytin-filled neuron; LY, Lucifer yellow-filled neurons.

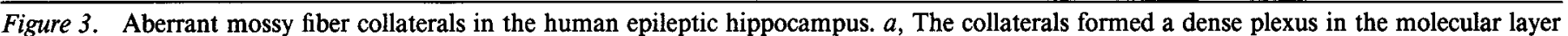

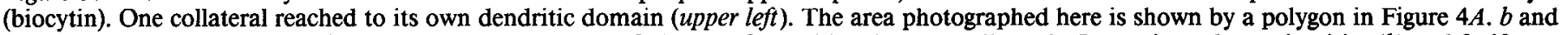

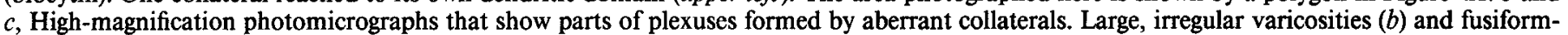

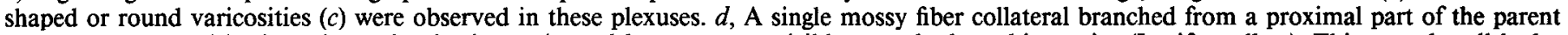

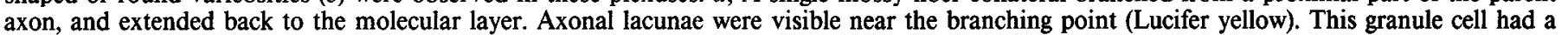
basal dendrite. Scale bar: $a, 17 \mu \mathrm{m} ; b, 7.5 \mu \mathrm{m} ; c, 10 \mu \mathrm{m} ; d, 30 \mu \mathrm{m}$. 

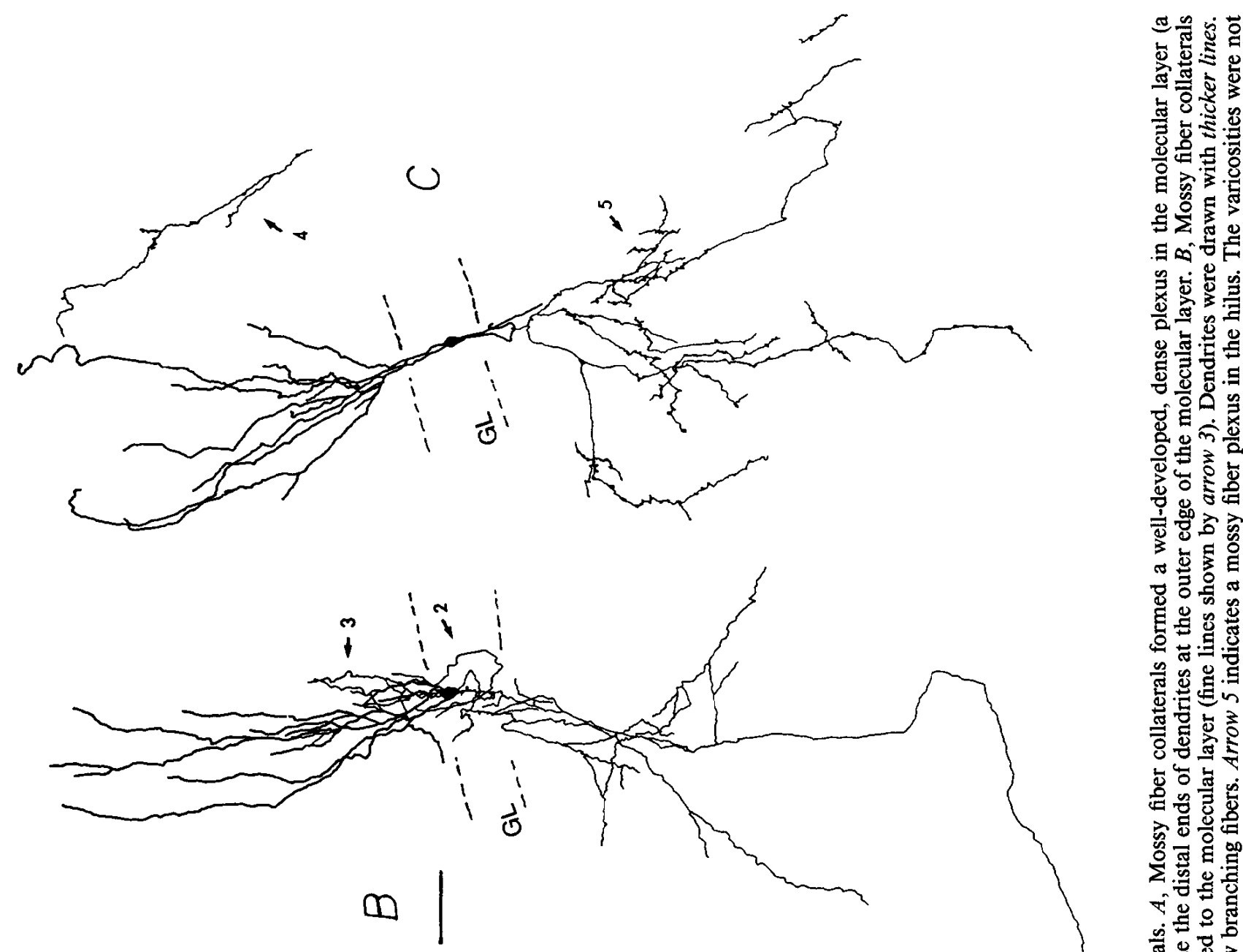

๙

E

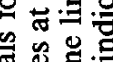

点的

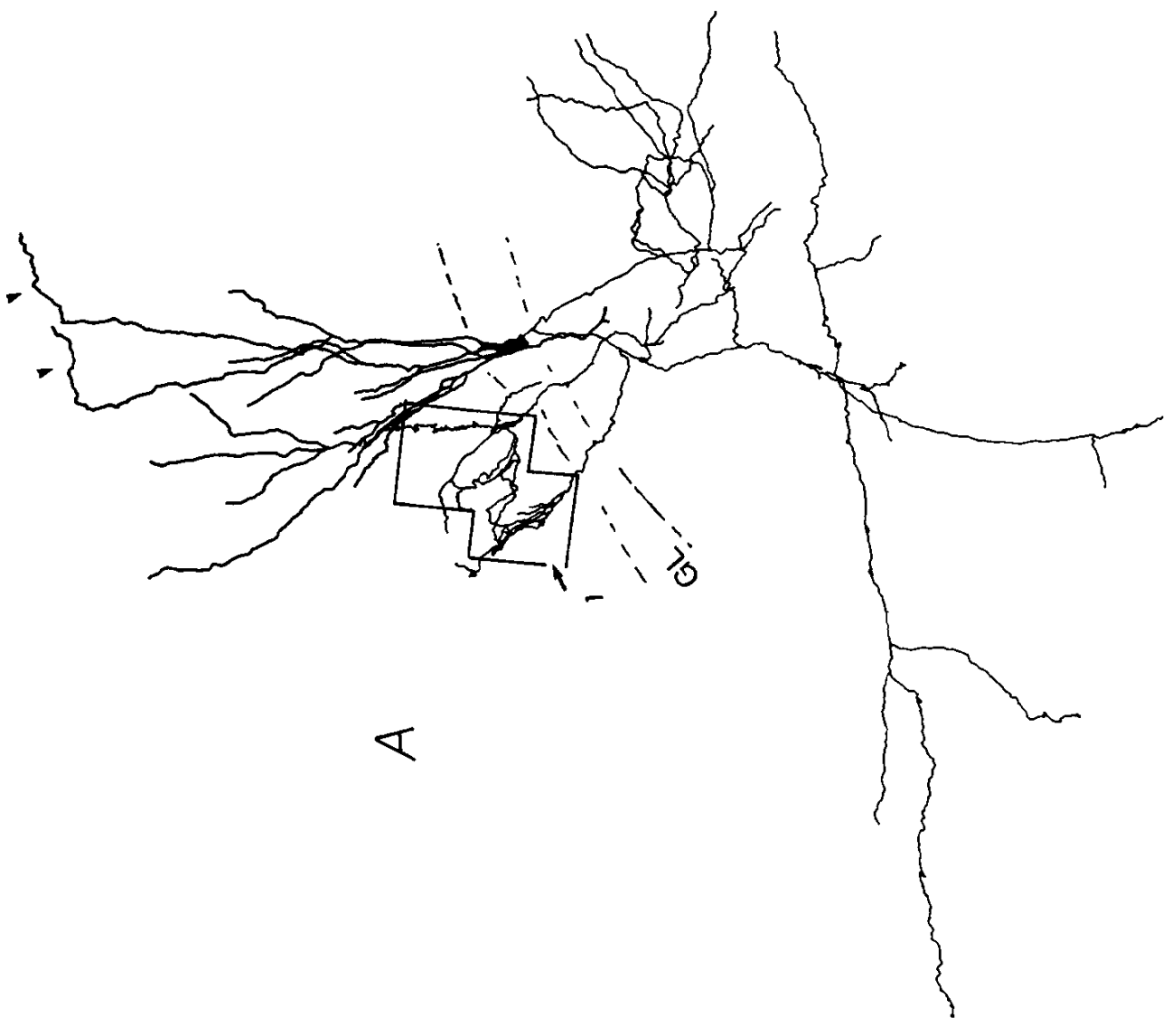

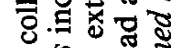

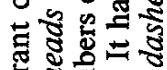

安家

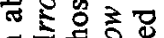

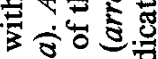

๑

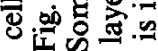

灵.토원

记

纪 응

응

옹. ․․

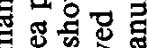

至的它

世男.

品岂可

的焉筞

긍요용

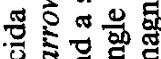

额兽宫

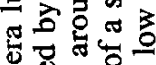

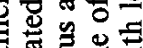

举运家

$\forall$ 的密。 
technique of staining only one cell at a time offered an advantage in showing that all the fibers stained in a given slice originated from a single neuron. This allowed us to perform qualitative and quantitative analyses of arborization patterns and domains of single mossy fiber axonal systems in human epileptic dentate granule cells, providing cellular evidence for aberrant mossy fiber paths and their detailed morphology.

Neurological and neuropathological conditions, examined in individual patients used in the present study, are discussed in relation to our data of intracellularly stained aberrant collaterals. This clinical information was made available from the CNP data base for the UCLA Epilepsy Surgery Program (see Table 3). Cell loss in the hippocampi and seizure histories of individual patients used in the present study were similar to those of previous reports on mossy fiber reorganization in human temporal lobe epilepsy (Sutula et al., 1989; Houser et al., 1990; Babb et al., 1991). In addition, the presence of aberrant mossy fiber collaterals was confirmed in the present patients by Timm's staining and/or dynorphin immunohistochemistry in comparison with autopsy control (Babb et al., 1991, 1992). These findings support our conclusion that the intracellularly stained aberrant axon collaterals in the granule/molecular layers in the present study probably represent trajectories of reorganized mossy fibers that are associated with medically intractable human temporal lobe epilepsy.

\section{Aberrant collaterals, cell loss, and seizure histories}

Animal studies demonstrated that extensive sprouting of the mossy fibers occurred in the supragranular layer following deafferentation of the dentate granule cells by the lesion of hilar projections (Laurberg and Zimmer, 1981). In human temporal lobe epilepsy, pathology often includes hilar cell loss. Table 3 summarizes the arborization patterns of the mossy fibers in 19 neurons in 10 patients in relation to seizure histories and selective cell loss in the dentate gyrus. A large variability of cell loss among patients was observed in the hilus $(5-75 \%$ in seven patients and no cell loss in two patients). In contrast, the cell loss was more consistent in the granule cell layer $(27-62 \%$ in nine patients). When the severity of cell loss was in the range of $50-75 \%$ in the hilus and $62 \%$ in the granule cell layer on average, most of the cells that were sampled for intracellular staining showed aberrant collaterals in the granule cell layer or in the molecular layer (patients $\mathrm{G}, \mathrm{H}$, and I). Considering an extremely low sampling probability of intracellular staining in a given hippocampal slice, this finding allowed us to estimate that a high proportion of the dentate granule cells could have aberrant collaterals in an advanced stage of cell loss in the present patient group. This estimate was supported by a report that these patients showed strong Timm's staining and/or positive immunoreactivity for dynorphin in the molecular layer (Babb et al., 1991, 1992; T. Babb, unpublished observations). Houser et al. (1990) also reported a positive correlation of the extent of aberrant collaterals to the severity of hilar cell loss. However, in cases where the degree of cell loss was less intense in the granule cell layer or in the hilus, we did not see any specific correlation between hilar cell loss and the presence of aberrant collaterals that were stained by our intracellular injection technique (Table 3 ). The lack of intracellularly stained aberrant collaterals in neurons of the specimens that showed less severe cell loss could reflect the small sample size in intracellular tracer injections. Further studies are needed to correlate the number of neurons that showed intracellularly stained aberrant collat-
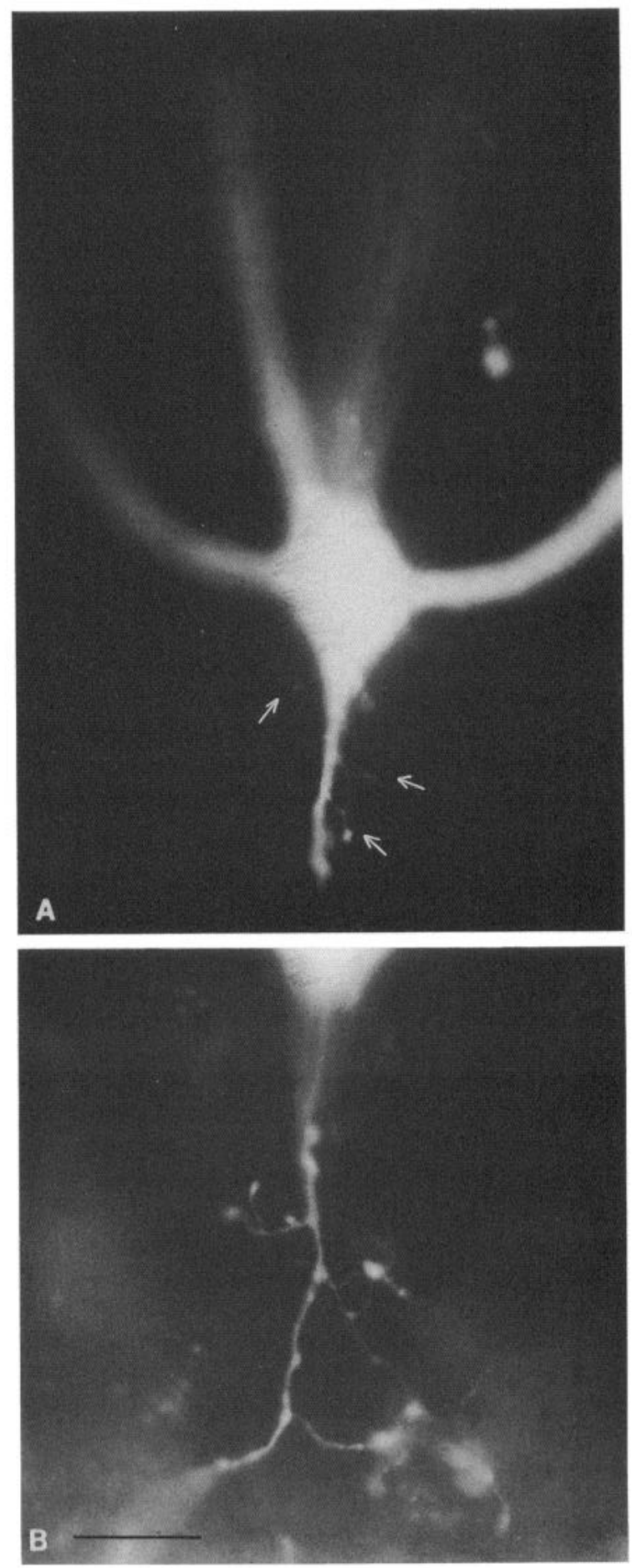

Figure 5. Filopodium-like fine processes were observed in the human dentate granule cells in epileptogenic hippocampi. They were present in the vicinity of the axon hillock (arrows in $A$ ) and in the parent axon immediately beneath the hillock $(B)$. Both pictures were taken from the same neuron stained by Lucifer yellow. Terminal enlargement was observed in one of these filopodia, which could be a putative growth cone (pointed by the bottom arrow in $A$ ). Scale bar: $A$ and $B, 10 \mu \mathrm{m}$.

erals and the amount of supragranular Timm's staining. In one case, it was possible to observe well-developed mossy fiber collaterals in the granule cell layer when cell loss was observed only in the granule cell layer, with no detectable cell loss in the hilus (patient $\mathrm{C}$ ). Although the type of neurons lost in the granule 
cell layer is not as well understood as in the hilus (deLanerolle et al., 1989; Sloviter et al., 1991), disruption of local circuits for the granule cells (Ribak et al., 1982) might potentiate the formation of supragranular mossy fibers.

Among the patients who showed aberrant collaterals in the present study, half of them (three of six) had histories of early childhood seizures. The clearest example of extensively developed aberrant collaterals in the supragranular layer was identified in a patient who had experienced tonic-clonic seizures since birth (patient $\mathrm{G}$ in Table 3). Thus, as reported by Houser et al. (1990), early childhood seizures contribute to mossy fiber collateralization. However, this observation was not generalized to the rest of the patients in the present study. This is because extended arbors of aberrant collaterals were found in only half of the present patients who had febrile seizures or early seizure onset. The rest of the patients experienced their first seizures at ages 13-31 even though the sampled neurons for intracellular injection in these patients showed extensively developed aberrant collaterals. We cannot rule out the possibility that these results reflect limited or biased sampling of intracellular staining. However, it appears that a history of early age seizures is not enough by itself to be considered as a determining factor of aberrant collateral formation.

\section{Varicosities, lacunae, and filopodia}

Intracellular injections of tracers revealed two types of varicosities along the fibers: small fusiform-shaped swellings and larger irregularly shaped expansions. Both were present in all 19 neurons regardless of the presence of aberrant fibers. Similar shapes of varicosities were observed in our control rat dentate granule cells, as well as in normal rat mossy fibers that were stained by the Golgi method (Blackstad and Kjaerheim, 1961) and by intracellular HRP injections (Claiborne et al., 1986). In humans, however, a previous Golgi study identified only irregularly shaped expansions as varicosities in normal autopsy hippocampus (Scheibel et al., 1974): fusiform-shaped swellings were reported as being pathological and unique to epileptic hippocampus. Scheibel et al. (1974) reported that fusiform-shaped swellings became enlarged and formed strings of beads in advanced pathological stages.

In addition to varicosities, there were lacunae in the present human specimens. As shown in Figures $1 f$ and $3 d$, the shape of lacunae was often round, and they were larger than varicosities and looked hollow inside. The contours of lacunae were not evenly stained but had one or a few densely stained spots. Some of them looked like abnormally swollen varicosities. It needs to be considered whether the lacunac are pathological. They resembled nodules in preterminal fibers of epileptic tissue. Their shapes and sizes were similar to pathological nodules described in Golgi-stained epileptic granule cells (Scheibel et al., 1974). Considering that our tissue is from epileptic patients, it is not surprising that we observed such pathologically deformed varicosities. On the other hand, formation of lacunae was reported to be a sign of rapid growth of the cyloplasm in developing human cerebral cortical neurons (Purpura, 1975). In these neurons, lacunae were often observed with filopodia, and they were considered as morphologic features of cell growth. Thus, lacunae may not be simply regarded as pathologically swollen varicosities. The determination of whether the lacunae are normally functioning synaptic varicosities needs to await electron microscopic study to verify the presence of a pre- and postsynaptic apparatus. It is beyond the scope of the present study to deter- mine when aberrant collaterals were formed and whether they are still in the process of growth. However, the presence of filopodium-like fine processes in the same mossy fibers that bear aberrant collaterals suggests that different degrees and stages of collateral formation might be present in our epileptic hippocampal specimens. An active regeneration of axonal collaterals might be an ongoing process in medically intractable epileptic human hippocampus.

\section{Arborization patterns of aberrant collaterals}

Aberrant mossy fiber collaterals were observed in the dentate granule cell layer and in the molecular layer in 9 of 19 total neurons stained in the present human specimens. The extent of arborization of the abcrrant collaterals in these nine neurons varied. Some of the neurons showed extensive plexuses, and others showed single collaterals with a few short branches. One possible explanation for this variability may be technical, perhaps due to insufficient transport of injected tracers. However, the fiber lengths that were stained by intracellular labeling averaged $3698.5 \mu \mathrm{m}$ in the present study (see Table 1). This measurement was $50 \%$ longer than the summed length of mossy fiber collaterals reported in the rat dentate granule cells $(2300$ $\mu \mathrm{m})$ (Claiborne et al., 1986). Thus, it is unlikely that our tracer transport was insufficient. Second, if the aberrant collaterals tended to have a smaller diameter compared to normally existing mossy fiber collaterals, they might not be satisfactorily stained. Indeed, some regenerated axon collaterals were reported to have smaller diameters in certain instances (Frotscher and Zimmer, 1983). However, in our case, the diameters of aberrant collaterals were not necessarily smaller than that of hilar collaterals. As shown in Figure 3, aberrant collaterals were sometimes larger in diameter than hilar axons. Third, the absence of aberrant collaterals in some neurons could result from a possibility that those collaterals were not in the same hippocampal slice in which the somata were located; that is, they could have been located more rostrally or caudally. This concern arose from reports on the rat indicating that hilar neurons projected to dentate granule cells at different levels in the anterior-posterior axis (Amaral and Insausti, 1989) and in the horizontal axis (Isokawa-Akesson and Finch, 1989). However, in contrast to hilar cells, mossy fibers in the rat hilus were reported to have a lamellar organization in which individual lamellae were confined within the thickness of the slices $(500 \mu \mathrm{m})$ (Claiborne, et al., 1986). Although this evidence does not rule out the possibility that human granule cell axons, especially aberrant collaterals, may travel substantially along the transversc axis, we have evidence that indirectly rejects this possibility. Our data demonstrate that the somata of neurons showing aberrant collaterals were significantly larger than neurons that did not show such collaterals. This suggests that an increased field of innervation and increased metabolic demands on the cell may produce somal hypertrophy (Grafstein and McQuarrie, 1978). Such an example has been reported in the regenerating trochlear nucleus in adult mammalian CNS (Murphy et al., 1990). Thus, if we had missed substantial numbers of aberrant collaterals that were hypothesized to be located outside of our slice specimens, we would not have found a positive correlation between enlarged somata and the presence of aberrant collaterals. Accordingly, we assume that most of the aberrant collaterals were observed in the present study. Based on this evidence, we conclude that our findings of variable amounts of aberrant mossy fiber col- 
laterals in the granule/molecular layers represent differing degrees of arborization.

Aberrant collaterals appear to form synapses. Electron microscopy has identified that zinc-containing terminals formed synapses on a dendrite of an unidentified neuron in human epileptic dentate gyrus (Babb et al., 1991). However, whether aberrant collaterals terminated on their own dendrites, on neighboring granule cell dendrites, or on inhibitory interneurons in the granule/molecular layers cannot be addressed in the present study. This is because we did not stain any target cells of those aberrant collaterals. In our study, in four of nine neurons that showed aberrant collaterals, their somata and proximal dendrites were surrounded by these collaterals and their plexuses. Furthermore, two neurons were found to have aberrant collaterals that reached their own dendritic domains and formed extensive plexuses. Whether these collaterals made synapses on their own dendrites, that is, autapses, could not be determined under light microscopic observations. However, in hippocampal culture, autapse formation has been reported in neurons grown by themselves in isolation (Bekkers and Stevens, 1991; Segal, 1991) and in neurons that were grown as a pair, also forming mutual synapses (C. Stevens, unpublished observations). This evidence suggests the possibility that dentate granule cells form presynaptic terminals on themselves through aberrant collaterals. On the other hand, a physiological study in an animal model of hippocampal epilepsy indicated a possibility that aberrant collaterals synapse on inhibitory interneurons in the dentate gyrus (Sloviter, 1992).

Studies in an experimentally produced aberrant mossy fiber system (Cronin et al., 1992) and in slice preparations from epileptic patients (Masukawa ct al., 1989) suggestcd that slightly decreased inhibition might be a mechanism for producing synchronous dentate gyrus activity. However, in human epileptic hippocampus, GABAergic neurons are preserved (Babb et al., 1989). During the depolarization of mossy fiber terminals for the release of glutamate, they corelease zinc, an antagonist of a subclass of excitatory amino acid receptors (Assaf and Chung, 1984; Howell et al., 1984), and dynorphin, an inhibitory modulator (Siggins et al., 1986; Terrian et al., 1988). Intracellular recording from epileptic dentate granule cells in humans showed that many neurons possess quite normal membrane properties and synaptic transmission (Isokawa et al., 1991). Further investigations are required to determine whether the excitatoryinhibitory balance of neuronal circuits in the dentate gyrus is permanently altered toward enhanced excitation in the anatomically reorganized, seizure-susceptible hippocampus.

\section{References}

Amaral DG, Insausti R (1990) The hippocampal formation. In: The human nervous system (Paxinos G, ed), pp 711-755. New York: Academic.

Assaf SY, Chung S (1984) Release of endogenous $\mathrm{Zn}^{2+}$ from brain tissue during activity. Nature 308:734-736.

Babb TL, Brown WJ, Pretorius J, Davenport C, Lieb JP, Crandall PII (1984) Temporal lobe volumetric cell densities in temporal lobe epilepsy. Epilepsia 25:729-740.

Babb IL, Pretorius JK, Kupfer W, Crandall PH (1989) Glutamate decarboxylase-immunoreactive neurons are preserved in human epileptic hippocampus. J Neurosci 9:2562-2574.

Babb TL, Kupfer WR, Pretorius JK, Crandall PH, Levesque MF (1991) Synaptic reorganization by mossy fibers in human epileptic fascia dentata. Neuroscience 42:351-363.

Babb TL, Pretorius JK, Kupfer WR, Mathern GW, Crandall PH, Levesque MF (1992) Aberrant synaptic reorganization in human epi- leptic hippocampus: evidence for feedforward excitation. Dendron 1:7-25.

Bekkers JM, Stevens CF (1991) Excitatory and inhibitory autaptic currents in isolated hippocampal neurons maintained in cell culture. Proc Natl Acad Sci USA 88:7834-7838.

Blackstad TW, Kjaerheim A (1961) Special axo-dendritic synapses in the hippocampal cortex: clcctron and light microscopic studies on the layer of mossy fibers. J Comp Neurol 117:133-159.

Claiborne BJ, Amaral DG, Cowan WM (1986) A light and electron microscopic analysis of the mossy fibers of the rat dentate gyrus. $J$ Comp Neurol 246:435-458.

Cronin J, Obenaus A, Houser CR, Dudek FE (1992) Electrophysiology of dentate granule cells after kainate-induced synaptic reorganization of the mossy fibers. Brain Res 573:305-310.

deLanerolle N, Kim JH, Robbins RJ, Spencer DD (1989) Hippocampal interneuron loss and plasticity in human temporal lobe epilepsy. Brain Res 495:387-395.

Desmond NL, Levy WB (1982) A quantitative anatomical study of the granule cell dendritic fields of the rat dentate gyrus using a novel probabilistic method. J Comp Neurol 212:131-145.

Engel J Jr (1987) Approaches to localization of the epileptogenic lesion In: Surgical treatment of the epilepsies (Engel J Jr, ed), pp 75-96. New York: Raven.

Frotscher M, Zimmer J (1983) Lesion-induced mossy fibers to the molecular layer of the rat fascia dentata: identification of postsynaptic granule cells by the Golgi-EM technique. J Comp Neurol 215:299311 .

Grafstein B, McQuarrie IG (1978) The role of the nerve cell body in axonal regeneration. In: Neuronal plasticity (Cotman $\mathrm{CW}$, ed), pp 155-195. New York: Raven.

Haas HL, Schaerer B, Vosmansky M (1979) A simple perfusion chamber for the study of nervous tissue slices in vitro. J Neurosci Methods 1:323-325.

Horikawa K, Armstrong WE (1988) A versatile means of intracellular labeling: injection of biocytin and its detection with avidin conjugates. J Neurosci Methods 25:1-11.

Houser CR ( 1990) Granule cell dispersion in the dentate gyrus of humans with temporal lobe epilepsy. Brain Res 535:195-204.

Houser CR, Miyashiro JE, Swartz BE, Walsh GO, Rich JR, DelgadoEscueta AV (1990) Altered patterns of dynorphin immunoreactivity suggest mossy fiber reorganization in human hippocampal epilepsy. J Neurosci 10:267-282.

Howell GA, Welch MG, Fredrichson CJ (1984) Stimulation induced uptake and release of zinc in hippocampal slices. Nature 325:522525.

Isokawa M, Levesque MF (1991) Increased NMDA responses and dendritic degeneration in human epileptic hippocampal neurons in slices. Neurosci Lett 132:212-216.

Isokawa M, Avanzini G, Finch DM, Babb TL, Levesque MF (1991) Physiologic properties of human dentate granule cells in slices prepared from epileptic patients. Epilepsy Res 9:242-250.

Isokawa-Akesson M, Finch D (1989) Neurophysiological characterization of commissurally projecting dentate neurons in the rat. Brain Res 480:92-104.

Laurberg S, Zimmer J (1981) Lesion-induced sprouting of hippocampal mossy fiber collaterals to the fascia dentata in developing and adult rats. J Comp Neurol 200:433-459.

Lorento de Nó R (1934) Studies on the structure of the cerebral cortex. II. continuation of the study of the ammonic system. J Psychol Neurol 46:113-177.

Masukawa LM, Higashima M, Kim JH, Spencer DD (1989) Epileptiform discharges evoked in hippocampal brain slices from epileptic patients. Brain Res 493:168-174.

Mello LEAM, Cavalheiro EA, Babb TL, Kupfer WR, Pretorius JK, Tan AM, Finch DM (1990) Pilocarpine-induced status epilepticus leads to chronic seizures and mossy fiber sprouting in the hippocampus. Epilepsia 31:675.

Murphy EH, Brown J, Lannuzzelli PG, Baker R (1990) Regeneration and soma size changes following axotomy of the trochlear nerve. $J$ Comp Neurol 292:524-536.

Purpura DP (1975) Dendritic differentiation in human cerebral cortex: normal and aberrant developmental patterns. In: Advances in neurology, Vol 12, physiology and pathology of dendrites (Kreutzberg GW, ed), pp 91-116. New York: Raven.

Ribak CE, Bradburne RM, Harris AB (1982) A preferential loss of 
GABAergic, symmetric synapses in epileptic foci: a quantitative ultrastructural analysis of monkcy ncocortex. J Neurosci 2:1725-1735.

Scheibel ME, Crandall PH, Scheibel AB (1974) The hippocampaldentate complex in temporal lobe epilepsy. Epilepsia 15:55-80.

Segal MM (1991) Epileptiform activity in microcultures containing one excitatory hippocampal neuron. J Neurophysiol 65:761-770.

Seress L, Mrzljak L (1987) Basal dendrites of granule cells are normal features of the fetal and adult dentate gyrus of both monkey and human hippocampal formations. Brain Res 405:169-174.

Seress L, Pokorny J (1981) Structure of the granular layer of the rat dentate gyrus: a light microscopic and Golgi study. J Anat 133:181195.

Seress L, Bakay RAE, Ribak CE (1991) Postsynaptic targets of Timmlabeled mossy fibers in rats and monkey hippocampus: a light and electron microscopic study. Soc Neurosci Abstr 17:127.

Siggins GR, Henriksen SJ, Chavkin C, Gruol D (1986) Opioid peptides and epileptogenesis in the limbic system: cellular mechanisms. In: Advances in neurology, Vol 44, Basic mechanisms of the epilepsies (Delgado-Escueta AV, Ward AA, Woodbury DM, Porter RJ, eds), pp 501-512. New York: Raven.

Sloviter RS (1992) Possible functional consequences of synaptic re- organization in the dentate gyrus of kainate-treated rats. Neurosci Lett 137:91-96.

Sloviter RS, Lossas AL, Barbaro NM, Laxer KD (1991) Calciumbinding protein (Calbindin-D28K) and parvalbumin immunocytochemistry in the normal and epileptic human hippocampus. J Comp Neurol 308:381-396.

Stewart W (1978) Intracellular marking of neurons with a highly fluorescent naphthalimide dye. Cell 14:741-759.

Sutula T, Xiao-Xian H, Cavazos J, Scott G (1988) Synaptic reorganization in the hippocampus induced by abnormal functional activity. Science 239:1147-1150.

Sutula T, Cascino G, Cavazos J, Parada I, Ramires L (1989) Mossy fiber synaptic reorganization in the epileptic human temporal lobe. Ann Neurol 26:321-330.

Tauck DL, Nadler JV (1985) Evidence of functional mossy fiber sprouting in hippocampal formation of kainic acid-treated rats. J Neurosci 5:1016-1022.

Terrian DM, Johnston D, Claiborne BJ, Ansah-Yiadom R, Strittmatter WJ, Rea MA (1988) Glutamate and dynorphin release from a subcellular fraction enriched in hippocampal mossy fiber synaptosomes. Brain Res Bull 21:343-351. 